\title{
Jersey calf performance in response to high-protein, high-fat liquid feeds with varied fatty acid profiles: Blood metabolites and liver gene expression
}

\author{
V. A. Swank, ${ }^{*}$ W. S. Bowen Yoho, † K. M. O’Diam, ${ }^{*}$ M. L. Eastridge, † A. J. Niehaus, $\ddagger$ and K. M. Daniels ${ }^{* 1}$ \\ *Department of Animal Sciences, Ohio Agricultural Research and Development Center, \\ †Department of Animal Sciences, and \\ łDepartment of Veterinary Clinical Sciences, The Ohio State University, Columbus, 43210
}

\begin{abstract}
Most available Jersey calf milk replacers (CMR) use edible lard as the primary fat source, which lacks medium-chain fatty acids (MCFA). However, Jersey cow milk consists of over $10 \%$ MCFA. The objective of this trial was to determine whether altering the fatty acid profile of CMR by increasing the amount of MCFA would alter liver lipid infiltration, liver gene expression, and blood metabolites when fed to Jersey calves. Fifty Jersey calves were fed 1 of 4 diets: pasteurized saleable whole milk (pSWM) from Jersey cows [27.9\% crude protein (CP), 33.5\% fat, dry matter (DM) basis]; CMR containing $100 \%$ of fat as edible lard (100:00; $29.3 \%$ CP, $29.1 \%$ fat, DM basis); CMR containing $20 \%$ of fat as coconut oil (CO; 80:20; $28.2 \%$ CP, $28.0 \%$ fat); or CMR containing $40 \%$ of fat as CO $(60: 40 ; 28.2 \%$ CP, $28.3 \%$ fat). Liquid diet DM intake averaged 0.523 , 0.500, 0.498, and $0.512 \mathrm{~kg} / \mathrm{d}$ for pSWM, 100:00, 80:20, and 60:40, respectively. Calves were fed their assigned liquid diet daily at 0600 and $1800 \mathrm{~h}$ from $2 \mathrm{~d}$ of age until 7 wk of age, and once daily until 8 wk of age. Calves were taken off trial at 9 wk of age. Calves had access to water and grain $(23.8 \% \mathrm{CP}, 2.71 \%$ fat, DM basis). Grain DM intake averaged 0.386, 0.439, 0.472, and $0.454 \mathrm{~kg} / \mathrm{d}$ for pSWM, 100:00, 80:20, and 60:40, respectively. Liver biopsy cores were obtained from 15 calves at $42 \mathrm{~d}$ of age (pSWM, $\mathrm{n}=4 ; 100: 00, \mathrm{n}=4$; $80: 20, \mathrm{n}=3 ; 60: 40, \mathrm{n}=4$ ) and from 4 baseline calves $<2 \mathrm{~d}$ of age. Liver biopsy cores were used for histological appraisal of lipid infiltration and gene expression analyses of short-, medium-, and long- chain acyl-coenzyme A dehydrogenases, sterol regulatory element binding transcription factor 1, acetyl coenzyme A carboxylase, and fatty acid synthase. Lipid infiltration and expression of selected genes were not different among diets. After an overnight fast, weekly blood samples were taken immediately before feeding at $0600 \mathrm{~h}$ via jugular
\end{abstract}

Received August 29, 2012.

Accepted March 4, 2013.

${ }^{1}$ Corresponding author: daniels.412@osu.edu venipuncture in all calves. Serum and plasma obtained from blood samples were used in the analyses of total protein, glucose, triglycerides, nonesterified fatty acids, and plasma urea nitrogen (PUN). Nonesterified fatty acids and PUN were the only blood metabolites affected solely by diet. Nonesterified fatty acids decreased in a linear manner with increased dietary CO inclusion. Calves fed pSWM had higher PUN than calves fed 80:20. In this trial, altering the fatty acid profile of CMR with the addition of medium-chain fatty acids from $\mathrm{CO}$ had minimal effects on liver lipid infiltration, liver gene expression, and blood metabolites when fed to Jersey calves.

Key words: fatty acid, Jersey calf, coconut oil, milk replacer

\section{INTRODUCTION}

Many Jersey calves are currently fed calf milk replacer (CMR) that is formulated to match the needs of Holstein calves. These products typically contain 20 to $28 \% \mathrm{CP}$ and $20 \%$ fat (DM basis). Inferior diets can negatively affect growth; for example, Bascom et al. (2007) observed that Jersey calves fed a 20\% CP, $20 \%$ fat (DM basis) CMR at 15\% of BW (adjusted weekly) had only minimal growth and the feeding method was not recommended. They also determined that the fat percentage of CMR intended for Jersey calves should be between 16 and 33\% (DM basis; Bascom et al., 2007).

In the United States, Jersey cow milk averages $4.69 \%$ fat (fluid basis), whereas Holstein cow milk averages only $3.65 \%$ fat (fluid basis; USDA-AIPL, 2011). In addition to the difference in milk fat percentage between Jersey and Holstein cows, milk from Jersey cows contains more medium-chain FA (MCFA) than does Holstein cow's milk. On an equal volume basis, milk fat from Jersey cows contains more C10:0 and C12:0 (both MCFA) when compared with milk fat from Holstein cows (Morales et al., 2000).

Currently, the most popular fat source used in CMR for both Jersey and Holstein calves is edible lard, which has a different FA profile than found in either Jersey 
or Holstein cow milk. Edible lard consists of long-chain FA (LCFA), and lacks both short-chain FA (SCFA) and MCFA. Coconut oil (CO), which consists of approximately $47 \%$ MCFA (Palmquist, 1988), may be an additional fat source in CMR formulations intended for Jersey calves.

Coconut oil has been formulated into CMR and used in prior experiments (Jenkins et al., 1985; Piot et al., 1999; Graulet et al., 2000; Mills et al., 2010), but not in CMR for Jersey calves. Mills et al. (2010) found evidence of fatty liver development when preweaned Holstein calves were fed a CMR (28\% CP, $23 \%$ fat; DM basis) containing $\mathrm{CO}$ with a total FA profile of $32 \%$ medium-chain triglycerides (TG). Mills et al. (2010) suspected that this type of fatty liver most likely represents the calf liver's inability to metabolize dietary fat in a timely manner.

Carbon chain length of dietary FA is important because it determines when and via which route the FA reaches the liver (Hocquette and Bauchart, 1999). The SCFA and MCFA up to 8 carbons in length can be absorbed in the abomasum, and MCFA up to 12 carbons in length can be absorbed in the small intestine. In these scenarios, NEFA first enter the portal vein, and then travel directly to the liver. In contrast, LCFA are absorbed into enterocytes, re-esterified into TG, and then incorporated into a chylomicron before release into the lymphatic system. Chylomicrons absorbed from the diet arrive at the liver after travel through the lymphatic system, arterial system, capillary beds, and venous system. Given the different routes, dietary SCFA and MCFA reach the liver faster in comparison to LCFA (Singh, 1997).

Because of the association between MCFA intake and liver metabolism, we studied Jersey calves fed liquid diets with varying amounts of MCFA from $\mathrm{CO}$ and assessed liver histology to evaluate degree of fat infiltration and liver gene activity. Genes of interest were short-chain acyl-CoA dehydrogenase (SCAD), medium-chain acyl-CoA dehydrogenase (MCAD), long-chain acyl-CoA dehydrogenase (LCAD), sterol regulatory element-binding transcription factor 1 (SREBF-1), fatty acid synthase (FASN), and acetylCoA carboxylase (ACACA). It was also of interest to study select blood metabolites involved with both lipid metabolism (NEFA and TG) and overall metabolism [glucose, plasma urea nitrogen (PUN), and total protein $(\mathbf{T P})$ ] to track pattern changes due to diet, time, or both.

The first hypothesis of this study was that feeding CMR diets containing 0, 20, or $40 \%$ of total fat as $\mathrm{CO}$ to Jersey calves would result in either linear or quadratic effects on lipid deposition in the liver, gene expression in the liver, and blood metabolites. The sec- ond hypothesis was that feeding a CMR diet containing $20 \%$ of total fat as CO to Jersey calves would result in similar lipid deposition in the liver, gene expression in the liver, and blood metabolites when compared with Jersey calves fed pasteurized Jersey saleable whole milk. Results of diet effects on DMI, total fat intake, individual FA intake, body growth, and health measures for these same animals are reported in detail in a companion paper (Bowen Yoho et al., 2013). Tables 1 to 3 summarize those data.

\section{MATERIALS AND METHODS}

\section{Animals and Treatments}

Animal procedures described herein were approved by The Ohio State University Institutional Animal Care and Use Committee (Protocol \#2010A00000186). Calves were enrolled in the study as they were born until a total of 50 calves was reached (male, $\mathrm{n}=18$; female, $\mathrm{n}=32$ ). Calves were individually housed and fed at the Waterman Dairy Farm at The Ohio State University. The trial was conducted from November 2010 to August 2011. A randomized complete block design with 4 treatments was used. Calves were blocked by sex, parity of dam, and date of birth, and assigned to 1 of 4 liquid feeds. One liquid feed was pasteurized $\left(72^{\circ} \mathrm{C}\right.$ for $\left.30 \mathrm{~s}\right)$ saleable whole milk (pSWM; n $=12$ ) from the Jersey herd at Waterman Dairy. The pSWM was $27.9 \%$ CP, $33.5 \%$ fat (DM basis). The other 3 liquid feeds were CMR varying in FA profile. These $3 \mathrm{CMR}$ were intended to be isonitrogenous and isocaloric, with dried whey, whey protein concentrate, dried whey product, dried skim milk, and dried milk protein included as protein sources. The CMR diets differed only in FA profile, which was a result of the fat sources used. Diet 100:00 $(\mathrm{n}=13)$ contained only edible lard as the fat source (Cow's Match-Jersey Blend, 29.3\% CP, 29.1\% fat (DM basis); Land O'Lakes Animal Milk Products, Shoreview, MN). Diet 80:20 ( $\mathrm{n}=13)$ contained edible lard (80\%) and CO (20\%) as fat sources (this was $28.2 \%$ CP, $28.0 \%$ fat; DM basis). Diet 60:40 $(\mathrm{n}=12)$ contained edible lard $(60 \%)$ and $\mathrm{CO}(40 \%)$ as fat sources (28.2\% CP, $28.3 \%$ fat; DM basis). All CMR diets were formulated and blended by Land O'Lakes Animal Products Company and were nonmedicated. Composition of diets is summarized in Table 1 . The CMR was reconstituted to $15 \%$ milk powder (ca. 13.8\% solids) and fed at 40.0 to $43.0^{\circ} \mathrm{C}$. Calves were fed their respective liquid diet twice daily (0600 and $1800 \mathrm{~h}$ ) from $2 \mathrm{~d}$ of age until $7 \mathrm{wk}$ of age, and once daily until weaning ( $8 \mathrm{wk}$ of age). During the first week of life, all calves were fed $1.9 \mathrm{~L}$ of liquid at each feeding. During wk 2 through 8 of life, calves were fed $2.27 \mathrm{~L}$ of liquid 
Table 1. Summary of feed composition data ${ }^{1}$

\begin{tabular}{|c|c|c|c|c|c|}
\hline \multirow[b]{2}{*}{ Variable } & \multirow[b]{2}{*}{ Grain } & \multirow[b]{2}{*}{$\mathrm{pSWM}^{1}$} & \multicolumn{3}{|c|}{ Milk replacer ${ }^{2}$} \\
\hline & & & 100:00 & $80: 20$ & $60: 40$ \\
\hline DM, \% & 94.9 & 14.1 & 92.4 & 91.8 & 92.0 \\
\hline $\mathrm{CP}, \%$ of $\mathrm{DM}$ & 23.8 & $27.9(3.70)$ & 29.3 & 28.2 & 28.2 \\
\hline Fat, $\%$ of DM & 2.71 & $33.5(4.72)$ & 29.1 & 28.0 & 28.3 \\
\hline \multicolumn{6}{|l|}{ FA, $\%$ of fat } \\
\hline $\mathrm{SCFA}^{3}$ & - & 3.66 & 1.67 & 1.82 & 1.75 \\
\hline $\mathrm{MCFA}^{4}$ & 2.87 & 12.54 & 1.26 & 10.14 & 18.31 \\
\hline $\mathrm{LCFA}^{5}$ & 94.44 & 83.52 & 94.66 & 85.55 & 77.73 \\
\hline Other & 3.17 & 0.34 & 2.46 & 2.43 & 2.16 \\
\hline
\end{tabular}

${ }^{1}$ Adapted from Bowen Yoho et al. (2013). Numbers in parentheses are on an as-fed basis and as reported by DHI; true protein values were determined by DHI and adjusted to a CP value assuming $6 \%$ nonprotein nitrogen (DePeters and Ferguson, 1992). pSWM = pasteurized saleable whole milk.

${ }^{2}$ 100:00 $=100 \%$ edible lard, 0\% coconut oil milk replacer (CMR; Cow's Match-Jersey Blend, Land O'Lakes Animal Milk Products, Shoreview, MN); 80:20 $=80 \%$ edible lard, 20\% coconut oil CMR; and 60:40 $=60 \%$ edible lard, $40 \%$ coconut oil CMR.

${ }^{3} \mathrm{SCFA}=$ short-chain FA $(4$ carbon FA).

${ }^{4} \mathrm{MCFA}=$ medium-chain FA (6-12 carbon FA).

${ }^{5} \mathrm{LCFA}=$ long-chain FA (sum of 14:0, 14:1, 15:0, 16:0, 16:1, 18:0, 18:1 trans, 18:1 cis, 18:2, 18:3, and 20:1).

at each feeding. Calves had ad libitum access to water at all times. In addition, all calves were given access to a common texturized grain $(23.8 \%$ CP, DM basis; AMPLI-Calf Jersey 22; Land O'Lakes Purina Feed LLC, St. Louis, MO). To avoid any confounding effects of grain on blood metabolite concentrations, calves were not offered grain on evenings preceding blood collection days (d 7, 14, 21, 28, 35, 42, 49, 56, and 63). Grain was offered the next morning following a single blood draw, except in the cases of $\mathrm{d} 7,21$, and 42 , when 4 blood samples were taken throughout a 12-h period and grain was withheld for a full $24 \mathrm{~h}$ for 34 calves (described later). Otherwise, calves had ad libitum access to grain.
Grain orts were recorded daily at the evening feeding and liquid feed orts (if any) were recorded at both feedings. Full details regarding animals, treatments, growth, and health data are reported in a companion paper (Bowen Yoho et al., 2013).

\section{Liver Biopsies and Analyses}

Liver Tissue Collection and Processing. Liver tissue biopsies were obtained from 15 calves $(\mathrm{n}=4$ each for pSWM, 100:0, and 60:40; $\mathrm{n}=3$ for 80:20) when they were approximately $42 \mathrm{~d}$ of age to assess degree of lipid infiltration and for gene expression stud-

Table 2. Summary of calf performance and feed intake data ${ }^{1}$

\begin{tabular}{|c|c|c|c|c|c|c|c|c|}
\hline \multirow[b]{2}{*}{ Variable $^{2}$} & \multicolumn{4}{|c|}{ Diet $^{3}$} & \multirow[b]{2}{*}{ SEM } & \multicolumn{3}{|c|}{ Contrast $^{4}$} \\
\hline & $\begin{array}{c}\text { pSWM } \\
(\mathrm{n}=12)\end{array}$ & $\begin{array}{c}100: 00 \\
(\mathrm{n}=13)\end{array}$ & $\begin{array}{c}80: 20 \\
(\mathrm{n}=13)\end{array}$ & $\begin{array}{c}60: 40 \\
(\mathrm{n}=12)\end{array}$ & & $\begin{array}{c}\text { pSMW } \\
\text { vs } 80: 20\end{array}$ & Linear & Quadratic \\
\hline Birth BW, kg & 26.8 & 26.8 & 26.5 & 26.4 & 0.9 & 0.82 & 0.74 & 0.95 \\
\hline Weaning BW, kg & 56.6 & 53.0 & 54.9 & 55.8 & 1.6 & 0.39 & 0.16 & 0.76 \\
\hline Weaning age, $\mathrm{d}$ & 58.2 & 57.3 & 57.0 & 57.3 & 0.8 & 0.33 & 0.99 & 0.75 \\
\hline Final BW, kg & 62.6 & 60.0 & 61.4 & 60.7 & 1.8 & 0.64 & 0.78 & 0.62 \\
\hline Liquid diet DMI, $\mathrm{kg} / \mathrm{d}$ & 0.523 & 0.500 & 0.498 & 0.512 & 0.006 & 0.01 & 0.20 & 0.35 \\
\hline Birth to weaning grain DMI, $\mathrm{kg} / \mathrm{d}$ & 1.63 & 1.78 & 1.76 & 1.74 & 0.12 & 0.46 & 0.83 & 0.98 \\
\hline Weaning to wk 9 grain DMI, $\mathrm{kg} / \mathrm{d}$ & 1.70 & 1.81 & 1.78 & 1.87 & 0.10 & 0.59 & 0.68 & 0.66 \\
\hline Grain DMI, kg/d & 0.386 & 0.439 & 0.472 & 0.454 & 0.080 & 0.42 & 0.89 & 0.78 \\
\hline Total DMI, kg/d & 0.909 & 0.938 & 0.970 & 0.966 & 0.067 & 0.49 & 0.76 & 0.81 \\
\hline
\end{tabular}

${ }^{1}$ Adapted from Bowen Yoho et al. (2013).

${ }^{2} \mathrm{ADG}=\mathrm{ADG}$ from birth to d 63 unless noted otherwise; gain:feed $=\mathrm{ADG} /$ total DMI.

${ }^{3}$ pSWM $=$ pasteurized saleable whole milk; 100:00 $=100 \%$ edible lard, $0 \%$ coconut oil milk replacer (CMR; Cow's Match-Jersey Blend, Land O'Lakes Animal Milk Products, Shoreview, MN); 80:20 = 80\% edible lard, 20\% coconut oil CMR; and 60:40=60\% edible lard, 40\% coconut oil CMR.

${ }^{4}$ pSWM vs. 80:20 = comparison of means of pSWM and 80:20; and linear and quadratic effects of coconut oil (0, 20\%, and 40\%). 
Table 3. Fat intakes from grain and liquid portion of the diets and total intakes of fat and FA during the entire trial for calves fed the 4 different liquid diets ${ }^{1}$

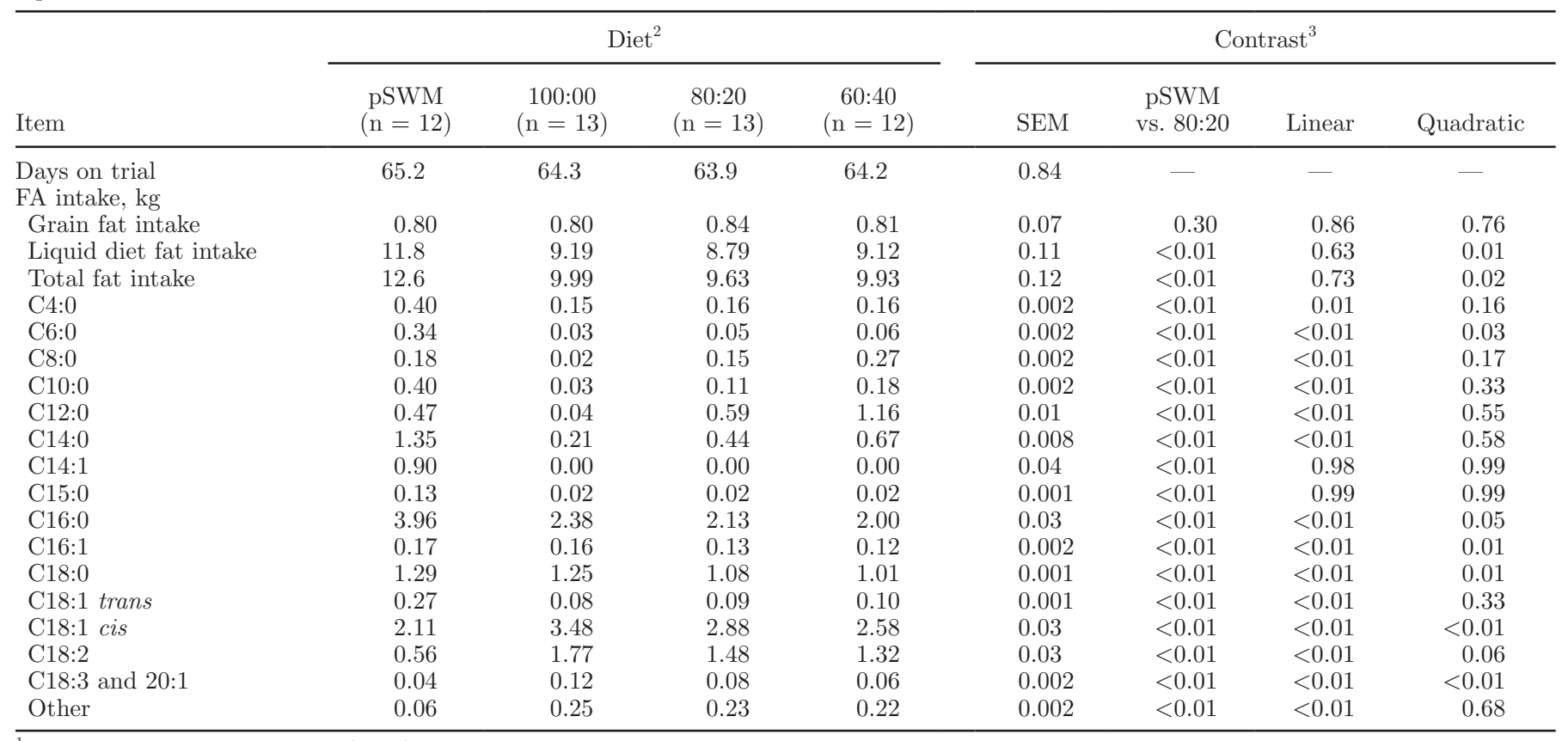

${ }^{1}$ Table 6 in Bowen Yoho et al. (2013).

${ }^{2} \mathrm{pSWM}=$ pasteurized saleable whole milk; 100:00 $=100 \%$ edible lard, $0 \%$ coconut oil milk replacer (CMR; Cow's Match-Jersey Blend, Land O'Lakes Animal Milk Products, Shoreview, MN); 80:20 = 80\% edible lard, 20\% coconut oil CMR; and 60:40=60\% edible lard, 40\% coconut oil CMR.

${ }^{3}$ pSWM vs. 80:20 = comparison of means of pSWM and 80:20; and linear and quadratic effects of coconut oil (0, 20\%, and 40\%).

ies. Baseline liver biopsies were also obtained from 4 male calves on $\mathrm{d} 2$ of life and processed identically to those obtained at $42 \mathrm{~d}$ of age. However, baseline calves were not enrolled in the study nor were they biopsied at $42 \mathrm{~d}$ of age. For biopsies, calves were taken from hutches to a nearby barn (within $25 \mathrm{~m}$ of hutch), and placed in a restraint chute. The liver biopsy procedure used was similar to the protocol described by Kahl et al. (1997). Briefly, the intended liver biopsy site was examined by ultrasound (Aloka model SSD-900V; Hitachi Aloka, Wallingford, CT; $3.5-\mathrm{MHz}$ sector probe) to ensure that the biopsy site was centered over liver tissue and to assess the thickness of the lateral body wall and locations of organs. The hair over the desired location on the calf's right side was clipped, and the area was scrubbed. Five milliliters of lidocaine (2\%) were infused to desensitize the skin, intercostal tissues, and peritoneum. A 1-cm incision was made through the skin. Transcutaneous biopsy samples were obtained $(\sim 100 \mathrm{mg} /$ sample, 5 samples) with an EZ Core Biopsy instrument (Products Group International, Lyons, CO; 16 gauge $1.6 \mathrm{~mm} \times 9 \mathrm{~cm}$ trocar or 14 gauge $2.1 \mathrm{~mm} \times$ $9 \mathrm{~cm}$ trocar). After liver biopsy samples were obtained, the puncture site was infused with procaine penicillin $(300,000$ units $/ \mathrm{mL})$ and the skin was sutured using $2-0$ polypropylene suture with 1 to 2 simple interrupted sutures. Sutures were removed 10 d later. For each calf, all but one liver biopsy core samples were snap frozen in liquid nitrogen and stored at $-80^{\circ} \mathrm{C}$ for later analysis. The remaining liver biopsy core was fixed in $10 \%$ formalin for $24 \mathrm{~h}$ and then placed in $70 \%$ ethanol until the time of embedding in paraffin.

Liver Histology. After embedding and cutting, the 5 - $\mu \mathrm{m}$ thick liver samples underwent general hematoxylin and eosin-Y staining. Stained slides were viewed on an Olympus IX81 microscope (Olympus Corporation, Shinjuku-ku, Tokyo, Japan) and 3 digital images per animal were captured at $320 \times$ magnification with an Olympus DP-72 camera operating in 24-bit RGB mode. Each image was centered on a central vein. Digital images were viewed by a single observer blinded to treatment. The observer was trained to look for signs of fatty liver and was to score each image as either positive or negative for lipid infiltration.

Liver Gene Expression. Snap frozen liver samples from each animal equaling up to $30 \mathrm{mg}$ were lysed using a TissueLyser LT (Qiagen, Valencia, CA). Each sample was placed in a $2-\mathrm{mL}$ microcentrifuge tube containing a $1.7-\mathrm{mm}$ stainless steel bead and $600 \mu \mathrm{L}$ of buffer RLT (Qiagen) and then lysed for $5 \mathrm{~min}$ at $50 \mathrm{~Hz}$. Tissue lysates were then processed using RNeasy mini kits (Qiagen) to extract total RNA from biopsy samples 
Table 4. Primer sequences ( $5^{\prime}$ to $3^{\prime}$ ) for quantitative reverse-transcription $\mathrm{PCR}^{1}$

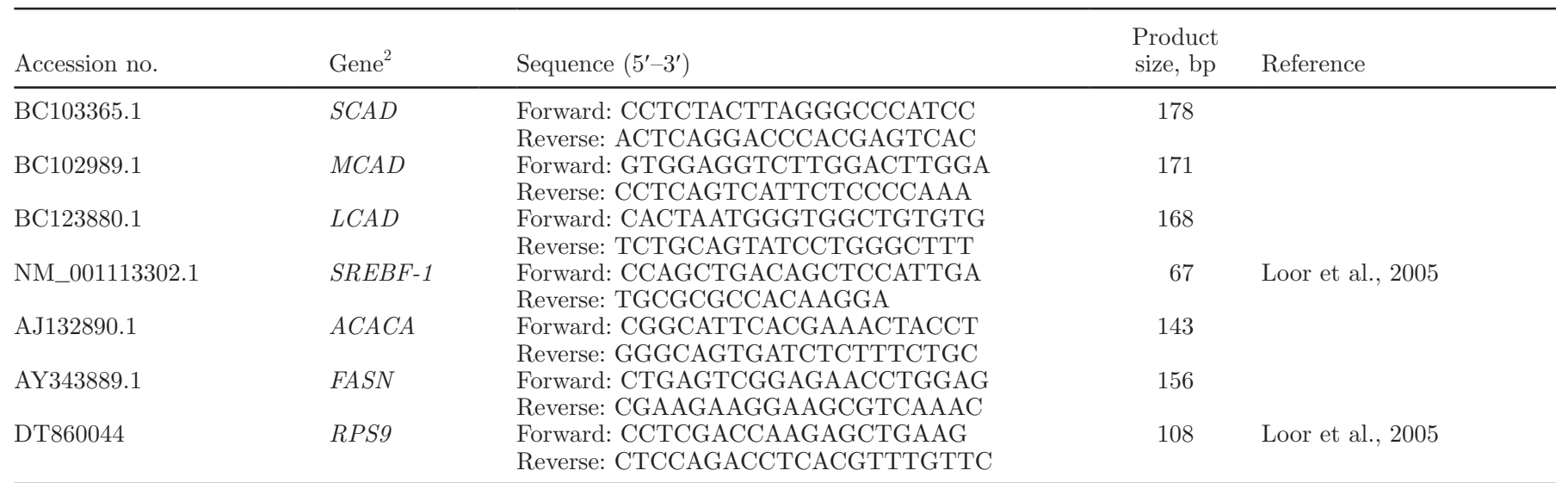

${ }^{1}$ Primer sequences not referenced were designed using Primer3 (v. 0.4.0; http://frodo.wi.mit.edu/).

${ }^{2} S C A D=$ short-chain acyl-CoA dehydrogenase; $M C A D=$ medium-chain acyl-CoA dehydrogenase; $L C A D=$ long-chain acyl-CoA dehydrogenase; $S R E B F-1=$ sterol regulatory element binding transcription factor $1 ; A C A C A=$ acetyl CoA carboxylase; FASN $=$ fatty acid synthase; RPS9 = ribosomal protein s9 (endogenous control).

using the procedures recently described by Rinaldi et al. (2010). The RNA quantity was determined using a NanoDrop ND-1000 spectrophotometer (NanoDrop Technologies, Rockland, DE); the 260 to 280 ratio averaged 2.06 for liver RNA.

Single-stranded cDNA was reverse transcribed from total RNA by denaturing $0.5 \mu \mathrm{g}$ of RNA in DNase/ RNase-free water, totaling $11.3 \mu \mathrm{L}$ at $70^{\circ} \mathrm{C}$ for $12 \mathrm{~min}$, and then placed on ice for $3 \mathrm{~min}$. Reverse transcription master mix $(8.7 \mu \mathrm{L})$ contained $4 \mu \mathrm{L}$ of $\mathrm{M}-\mathrm{MLV}$ RT $5 \times$ buffer (Promega, Madison, WI), $2 \mu \mathrm{L}$ of $0.1 M$ dithiothreitol, $1 \mu \mathrm{L}$ of $10 \mathrm{mM}$ dNTP mix (Promega), $1 \mu \mathrm{L}$ of oglio(dT)15 primer, $0.5 \mu \mathrm{L}$ M-MLV reverse transcriptase (Promega), and $0.2 \mu \mathrm{L}$ of RNase inhibitor (Promega). The entire volume of master mix was added to each well of denatured RNA in a 96 well PCR plate for a final reaction volume of $20 \mu \mathrm{L}$ (Bio-Rad, Hercules, CA). Reverse transcription was carried out in an iQ5 Multicolor reverse transcription PCR Detection System (Bio-Rad) with cycle conditions at $40^{\circ} \mathrm{C}$ for $1 \mathrm{~h}$, followed by $95^{\circ} \mathrm{C}$ for $10 \mathrm{~min}$. Resultant cDNA was then diluted 1:1 with addition of $20 \mu \mathrm{L}$ of DNase/RNase-free water. The cDNA was stored at $-20^{\circ} \mathrm{C}$ until further use.

Primers were designed with Primer3 (v. 0.4.0; http:// frodo.wi.mit.edu/) for genes SCAD, MCAD, LCAD, ACACA, and FASN (Table 4). Primer sequences for SREBF-1 and the endogenous control gene, ribosomal protein s9, were the same as in Loor et al. (2005; Table 4). Primer efficiencies were determined for each primer pair by the use of serial dilutions of cDNA, using the following equation: percent efficiency $=(10-1 /$ slope $-1) \times 100$. Primer efficiencies averaged $102.1 \%$.

Quantitative reverse transcription PCR (qPCR) reactions were carried out using a master mix consist- ing of $10.5 \mu \mathrm{L}$ of RNase/Dnase-free water, $12.5 \mu \mathrm{L}$ of iQ SYBR Green Supermix (Bio-Rad), and $0.5 \mu \mathrm{L}$ of each respective forward and reverse primer. Twentyfour microliters of master mix were then added to 2 $\mu \mathrm{L}$ of first-strand cDNA, yielding a $26 \mu \mathrm{L}$ reaction volume for $\mathrm{qPCR}$. Amplification was carried out in an iQ5 Multicolor Real-Time PCR Detection System (Bio-Rad) with conditions set at 1 cycle at $95^{\circ} \mathrm{C}$ for 5 min, 40 repeating cycles at $95^{\circ} \mathrm{C}$ for $10 \mathrm{~s}$, and then at $57.4^{\circ} \mathrm{C}$ for $45 \mathrm{~s}$. Negative assay controls included no template cDNA controls and no reverse transcriptase controls. Immediately after the $\mathrm{qPCR}$ experiment, a melt curve analysis was conducted to verify the purity of amplicons and absence of primer dimers. For this, plate temperature was increased from 65 to $95^{\circ} \mathrm{C}$ in $1^{\circ} \mathrm{C}$ increments.

After qPCR, representative samples of PCR products for each primer pair were electrophoresed on a $2 \%$ agarose gel to confirm purity and size of each amplicon. All PCR products were single bands that corresponded with predicted product size (data not shown).

Relative mRNA abundance of target genes is expressed as the fold differences of target genes relative to the expression of the endogenous reference gene ribosomal protein s9 and baseline animals. Specifically, relative mRNA abundance for each target gene was determined by first subtracting the cycles to threshold (Ct) value of the endogenous reference gene from the $\mathrm{Ct}$ value for each target gene, thus yielding $\Delta \mathrm{Ct}$ values. Next, $\Delta \mathrm{Ct}$ values for baseline animals $(\mathrm{n}=3$; all data from one baseline animal were discarded on the basis of nonconformity for all genes) were averaged, resulting in a $\Delta \mathrm{Ct}_{\text {baseline average for each target gene; }}$ $\Delta \mathrm{Ct}_{\text {baseline average }}$ was subtracted from $\Delta \mathrm{Ct}_{\text {target gene }}$ values for all other observations, yielding $\Delta \Delta \mathrm{Ct}$ values. For 
ease of interpretation, data are presented as $2^{(-\Delta \Delta \mathrm{Ct})}$ values.

\section{Blood Collection and Analysis}

Weekly Blood Collection and Processing. All calves were bled weekly $(0,1,2,3,4,5,6,7,8$, and 9 wk of life) at $0600 \mathrm{~h}$ (after an overnight fast) to track changes in blood metabolite concentrations over time, by diet, or both. Three vials of blood (total volume of blood collected $\leq 26 \mathrm{~mL}$ ) were obtained via jugular venipuncture at each time point into evacuated tubes, 2 tubes with no anticoagulant (serum tubes), and 1 tube with sodium fluoride (plasma tube). All samples were kept on ice after collection and transported to a laboratory within $1 \mathrm{~h}$ for further processing. A subset of animals on each treatment (pSWM, $\mathrm{n}=8 ; 100: 00$, $\mathrm{n}=9 ; 80: 20, \mathrm{n}=9 ; 60: 40, \mathrm{n}=8)$ underwent more intensive blood sampling at 1,3 , and $6 \mathrm{wk}$ of life to track pattern changes in metabolite concentrations in relation to feeding and age. In these selected animals, 4 blood collection incidents (totaling no more than 26 $\mathrm{mL}$ of blood per incident; same size and type of tubes as used in $0600 \mathrm{~h}$ sample collection) took place at 0600 (fasted sample), 0800, 1200, and $1800 \mathrm{~h}$ (results not shown).

Blood Metabolite Analyses. Plasma was used for analysis of glucose and PUN; serum was used for analysis of TP, NEFA, and TG. All blood samples were processed and analyzed using methods similar to Daniels et al. (2008) for glucose, PUN, TP, and TG. The HR Series NEFA (2) reagent set (Wako Pure Chemicals Industries Ltd., Richmond, VA) was used to determine NEFA concentrations of serum. An aqueous NEFA standard was used for this assay $(1.0 \mathrm{mmol} / \mathrm{L}$; Wako Pure Chemicals Industries Ltd.). Concentrations were determined at $550 \mathrm{~nm}$ using an ELX 808 Ultra Microplate Reader (Bio-Tek Instruments Inc., Winooski, VT). Intra- and interassay CV for all metabolite assays averaged 1 and $3 \%$, respectively.

\section{Statistical Analyses}

Histology data for liver biopsy samples that underwent hematoxylin and eosin staining were not analyzed statistically, as no lipid infiltration was noted in any samples. All other data were analyzed using SAS (SAS Version 9.1.3, SAS Institute Inc., Cary, NC).

Prior to ANOVA, normality of data distribution for each treatment and gene combination in the qPCR data set were examined using the Shapiro-Wilk test in the univariate procedure of SAS. The null hypothesis that data were from a random sample from a normal distribution was rejected when $P \leq 0.05$; this occurred in 3 instances. Because of that, a more conservative $\alpha$ was selected for later ANOVA testing of qPCR data; differences were declared significant at $P \leq 0.02$. Additionally, absence of heterogeneity of variance was confirmed with Hartley F-max testing (5 variances, 2 df, $0.05 \alpha$ ). The qPCR data were analyzed using the Mixed Procedure of SAS; data submitted to SAS consisted of $2^{(-\Delta \Delta \mathrm{Ct})}$ values. Treatment was the single fixed effect in the model, and the random effect was calf within treatment.

Weekly blood metabolite data were analyzed using the mixed procedure of SAS with repeated measures. The repeated measure was week and the subject used in tests was block; a compound symmetry covariance structure was specified for all tests. No denominator degrees of freedom approximation was specified. Preplanned, nonorthogonal contrast statements were used to examine diet differences, when present: pSWM versus 80:20; linear effect of $\mathrm{CO}(0,20$, and $40 \%)$; and quadratic effect of CO $(0,20$, and $40 \%)$. Orthogonal polynomial contrasts were used to test for linear and quadratic responses over time. All data are reported as $\mathrm{LSM} \pm \mathrm{SE}$ of the mean. Significance was declared at $P \leq 0.05$ except for in qPCR testing, as noted above.

\section{RESULTS AND DISCUSSION}

\section{Intake and Performance}

Calf intake and performance data are described in detail in a companion paper by Bowen Yoho et al. (2013). Briefly, initial and final BW did not differ by diet, nor did weaning age. Calves fed pSWM compared with 80:20 had a higher liquid diet DMI (0.523 vs. $0.498 \mathrm{~kg} / \mathrm{d}$, respectively) and similar grain DMI (0.386 vs. $0.472 \mathrm{~kg} / \mathrm{d}$, respectively). Coconut oil inclusion did not affect liquid diet DMI, grain DMI, or total DMI. Fat intake from grain did not differ between diets. Differences in liquid diet fat intake were observed. Calves fed pSWM consumed $11.8 \mathrm{~kg}$ of fat from the liquid diet; whereas calves fed 80:20 consumed only $8.79 \mathrm{~kg}$ of fat from the liquid diet $(P<0.01)$. A quadratic effect of $\mathrm{CO}$ on consumption of fat from the liquid portion of the $\operatorname{diet}(P=0.01)$ was also noted; liquid diet fat consumption was $9.19,8.79$, and $9.12 \mathrm{~kg}$ for 100:00, 80:20, and 60:40, respectively. Total fat intake was higher in calves fed pSWM than in calves fed 80:20 (12.6 vs. $9.63 \mathrm{~kg}$, respectively). Coconut oil inclusion had a quadratic, but not linear, effect on total fat intake. Total fat intakes were $9.99,9.63$, and $9.93 \mathrm{~kg}$ for 100:00, 80:20, and 60:40, respectively.

Individual FA intake differed by diet in several instances. Most relevant to the current study is intake of MCFA. Intake of C6:0, C8:0, and C10:0 were higher in 


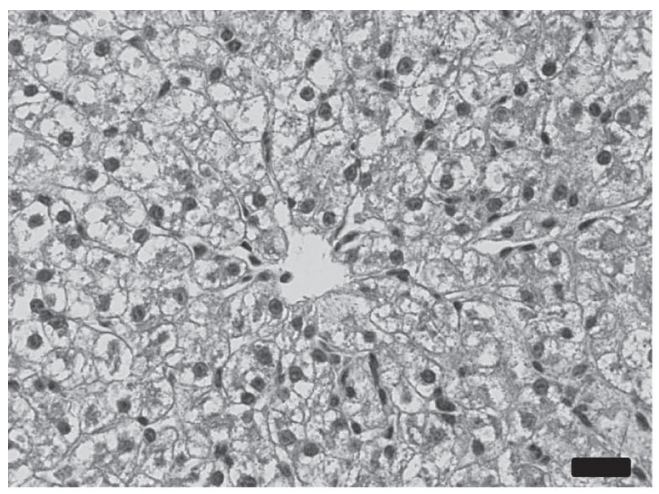

Baseline

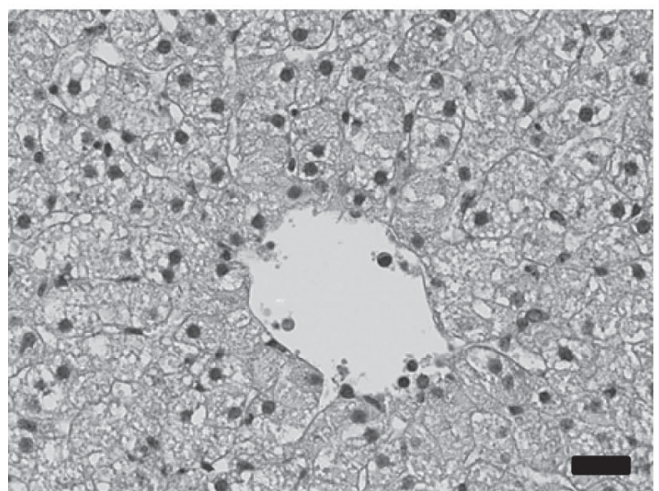

100:00

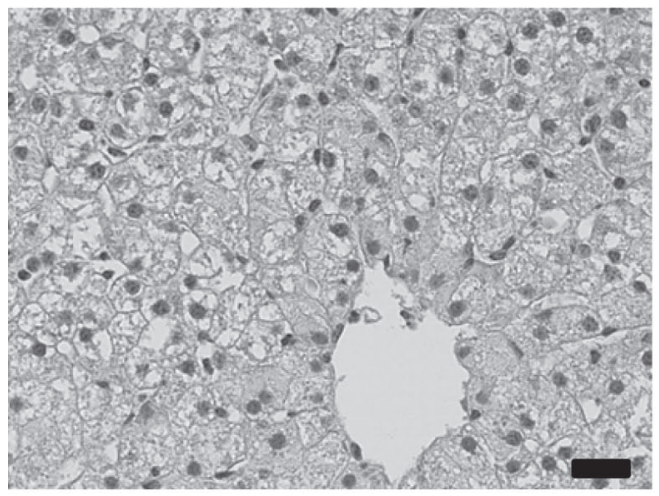

60:40

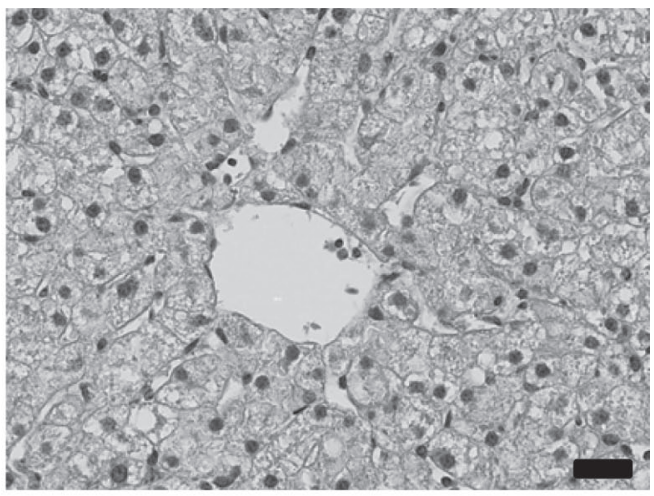

pSWM

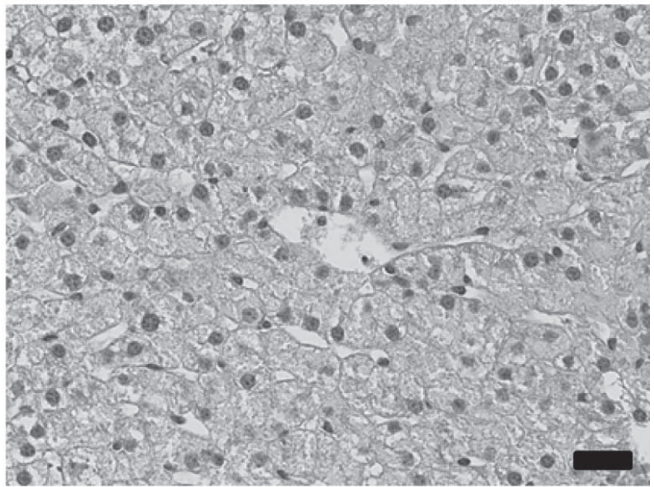

$80: 20$

Figure 1. Representative hematoxylin and eosin stained liver biopsies. Baseline samples obtained at $2 \mathrm{~d}$ of age, all other samples obtained at $42 \mathrm{~d}$ of age. Diets were pasteurized saleable whole milk (pSWM; $27.9 \%$ CP, 33.5\% fat, DM basis); calf milk replacer (CMR; Cow's MatchJersey Blend, Land O'Lakes Animal Milk Products, Shoreview, MN) containing 100\% of fat as edible lard (100:00; 29.3\% CP, 29.1\% fat); CMR containing $20 \%$ of fat as coconut oil (CO; $80: 20 ; 28.2 \%$ CP, $28.0 \%$ fat); and CMR containing $40 \%$ of fat as CO (60:40; $28.2 \%$ CP, $28.3 \%$ fat). No difference in lipid infiltration of the liver was noted by visual appraisal. Scale bar $=20 \mu \mathrm{m}$.

calves fed pSWM compared with those fed 80:20 (0.92 vs. $0.31 \mathrm{~kg}$, respectively); calves fed 80:20, however, had a higher intake of C12:0 compared with calves fed pSWM (0.59 vs. $0.47 \mathrm{~kg}$, respectively). Overall, calves fed pSWM had a higher intake of MCFA than calves fed 80:20. In addition, a linear increase in total MCFA consumption with $\mathrm{CO}$ inclusion in CMR was observed (Bowen Yoho et al., 2013).

\section{Liver Histology}

One objective of this trial was to examine the effects of $\mathrm{CO}$ inclusion in CMR on FA metabolism in Jersey calves. Hematoxylin and eosin stained liver samples showed no noticeable difference in the amount of fat present by diet or when visually compared with baseline animals. Representative samples from each 
diet plus the baseline are presented in Figure 1. Given that observation, increasing the amount of $\mathrm{CO}$ in the diet did not appear to increase the amount of lipid infiltration in the liver. An increase in the amount of lipid infiltration would have most likely indicated that the calf's hepatocytes had reduced FA oxidation or increased incorporation of FA into TG in excess of what was being exported to peripheral tissues. Coconut oil included in CMR as the sole source of fat has been shown to increase the amount of TG present in the liver of Holstein-Friesian calves when fed for $19 \mathrm{~d}$; TG content of wet liver weight was determined to be $4.1 \%$ compared with $0.2 \%$ for those not fed CO (Piot et al., 1999). Piot et al. (1999) showed that CO fed at high levels can increase lipid infiltration in the liver. However, even at the highest level of $\mathrm{CO}$ inclusion in this trial ( $40 \%$ of total fat), no increase in liver lipid infiltration was seen. Another study revealed that feeding a CMR containing 32\% medium-chain TG from CO to Holstein calves also led to increased liver lipid infiltration; TG content was $6.5 \%$ of wet liver weight for those fed CO compared with $5.3 \%$ for those fed CMR that used animal fat as the sole fat source (Mills et al., 2010). The highest percentage of MCFA intake on this trial was $18.3 \%$ for diet $60: 40$ (Table 3 ); this percentage is just over half of the amount fed in Mills et al. (2010). It seems that neither $\mathrm{CO}$ nor MCFA were included at a high enough level in CMR to elicit an increase in liver lipid infiltration in this trial.

\section{Liver Gene Expression}

A second objective was to test whether $\mathrm{CO}$ inclusion alters a calf's metabolism is through examination of gene expression associated with FA metabolism. Fatty acid metabolism in the liver consists of 3 main processes: FA oxidation, FA synthesis and TG assembly.

Fatty acid oxidation in the liver occurs mainly through $\beta$-oxidation, where FA in mitochondria are either completely oxidized to acetyl-CoA or partially oxidized to ketones. Once inside the mitochondrion, carbon chain-length specific enzymes, known as acyl-CoA dehydrogenases, catalyze the next step of $\beta$-oxidation. Specifically, 3 members of this family are involved in $\beta$-oxidation of FA: SCAD acts on acyl-CoA with carbon chain lengths of 4 to 6 (Ikeda et al., 1985); MCAD will act on acyl-CoA with carbon chain lengths of 4 to 16, with preferred substrates that have carbon chain lengths of 6 to 12 (Zeng and Li, 2005); and LCAD acts on acyl-CoA with carbon chain lengths of 6 to 16, with preferred substrates that have carbon chain lengths of 8 to 14 (Ikeda et al., 1985). In this study, CMR with 20 and $40 \% \mathrm{CO}$ inclusion had FA profiles that contained larger amounts of MCFA than did the CMR containing only edible lard. For this reason, it can be assumed that calves fed diets with greater amounts of MCFA absorbed more intact MCFA. Increased $\beta$-oxidation of MCFA would have been a potential adaptive change in lipid metabolism in the liver to deal with increased intake of MCFA. However, no differences in gene expression by diet for SCAD, MCAD, or LCAD were observed in this trial (Table 5).

Fatty acid synthesis and TG assembly both occur in the liver. Fatty acid synthase is involved with de novo FA synthesis (Strable and Ntambi, 2010). If FA are not oxidized in liver, they can undergo TG assembly. In this case, FA are esterified onto glycerol to form TG and are normally transported out of the liver for storage or use in peripheral tissues. Sterol regulatory element-binding transcription factor 1 is at least partially responsible for regulating FA synthesis prior to FA esterification onto glycerol (McFadden and Corl, 2009). The lipid may pool in hepatocytes unless FA are oxidized or TG are exported as very low density lipoproteins (Nguyen et al., 2008). Graulet et al. (2000) noted that 15-dold Holstein $\times$ Friesian calves fed CMR (22.4\% fat) that contained $47 \%$ MCFA as total fat for $19 \mathrm{~d}$ had increased hepatic TG formation, but noted no increase in very low density lipoprotein production. As a result, these calves developed fatty liver (Graulet et al., 2000). Acetyl-CoA carboxylase catalyzes the synthesis of malonyl-CoA, which donates 2 carbons per cycle during de novo synthesis of FA; malonyl-CoA also inhibits mitochondrial oxidation of FA (Strable and Ntambi, 2010). Our analysis of liver gene expression revealed no diet differences in expression of genes associated with FA synthesis, namely SREBF-1, ACACA, and FASN (Table 5). This goes along with our histology findings.

Based on the relative expression of tested genes, those associated with $\beta$-oxidation (SCAD, MCAD, and LCAD) were more abundant at the time of biopsy in 42-d-old Jersey calves than those associated with FA synthesis (SREBF-1, ACACA, and FASN; Table 5). Data also suggest that, numerically, baseline animals had relatively higher expression of SREBF-1 and ACACA than 42-d-old Jersey calves; whereas the opposite was true of SCAD, MCAD, and LCAD (Table 5).

Potential downfalls were noted in this study with regard to determination of gene expression in the liver. First, liver biopsy collection time in relation to feeding may have been an issue. Biopsies were performed approximately $6 \mathrm{~h}$ after morning feeding, which may have been too long after a feeding to observe differences in liver gene expression. Second, a relatively small number of animals per treatment were biopsied, leading to low statistical power. Another potential downfall is that only gene expression was analyzed here, rather than protein abundance or enzyme activity related to these 
Table 5. Relative mRNA abundance of selected genes in liver biopsies from 42-d-old Jersey calves fed 1 of 4 $\operatorname{diets}^{1}$

\begin{tabular}{|c|c|c|c|c|c|c|}
\hline \multirow[b]{2}{*}{ Gene $^{2}$} & \multicolumn{4}{|c|}{ Treatment $^{3}$} & \multirow[b]{2}{*}{$\begin{array}{c}\text { SEM } \\
(\mathrm{n}=3)\end{array}$} & \multirow[b]{2}{*}{$P$-value ${ }^{4}$} \\
\hline & $\begin{array}{l}\mathrm{pSWM} \\
(\mathrm{n}=4)\end{array}$ & $\begin{array}{c}100: 00 \\
(\mathrm{n}=4)\end{array}$ & $\begin{array}{c}80: 20 \\
(\mathrm{n}=3)\end{array}$ & $\begin{array}{c}60: 40 \\
(\mathrm{n}=4)\end{array}$ & & \\
\hline \multicolumn{7}{|l|}{$\beta$-oxidation } \\
\hline$S C A D$ & 2.906 & 2.756 & 3.646 & 5.771 & 1.660 & 0.457 \\
\hline$M C A D$ & 2.462 & 3.697 & 3.425 & 2.537 & 0.999 & 0.689 \\
\hline$L C A D$ & 3.253 & 3.542 & 2.998 & 5.008 & 1.463 & 0.705 \\
\hline \multicolumn{7}{|c|}{ FA synthesis } \\
\hline$S R E B F-1$ & 0.738 & 0.279 & 0.246 & 0.438 & 0.237 & 0.379 \\
\hline$A C A C A$ & 0.649 & 0.214 & 0.891 & 0.311 & 0.225 & 0.143 \\
\hline FASN & 1.517 & 0.426 & 1.534 & 0.575 & 0.811 & 0.588 \\
\hline
\end{tabular}

${ }^{1}$ Data are expressed as $2^{-\Delta \Delta \mathrm{Ct}}$ values; numbers are fold differences in gene expression in relation to baseline samples obtained at $2 \mathrm{~d}$ of age (e.g., $2.906=2.906$-fold more mRNA than baseline).

${ }^{2} S C A D=$ short-chain acyl-CoA dehydrogenase; $M C A D=$ medium-chain acyl-CoA dehydrogenase; $L C A D=$ long-chain acyl-CoA dehydrogenase; $S R E B F-1=$ sterol regulatory element binding transcription factor 1 ; $A C A C A=$ acetyl $\mathrm{CoA}$ carboxylase; $F A S N=$ fatty acid synthase.

${ }^{3}$ Treatments were pasteurized saleable whole milk (pSWM; $27.9 \%$ CP, $33.5 \%$ fat, DM basis); calf milk replacer (CMR; Cow's Match-Jersey Blend, Land O'Lakes Animal Milk Products, Shoreview, MN) containing $100 \%$ of fat as edible lard (100:00; $29.3 \%$ CP, $29.1 \%$ fat): CMR containing 20\% of total fat as coconut oil (CO; 80:20; $28.2 \% \mathrm{CP}, 28.0 \%$ fat); CMR containing $40 \%$ of total fat as CO (60:40; $28.2 \% \mathrm{CP}, 28.3 \%$ fat).

${ }^{4}$ Significance declared when $P \leq 0.02$.

genes of interest. It is feasible that post-transcriptional changes in these genes occur that may ultimately affect either protein production or function or both.

\section{Blood Metabolites}

Fasting serum TP concentration was not affected by the interaction of diet and week, the main effect of diet, or the main effect of week, and averaged $5.04 \mathrm{~g} /$ $\mathrm{dL}$ across all diets and weeks (data not shown). This is in agreement with research done by Lee et al. (2009) where TP concentration in Holstein calves fed SWM was not different from those fed CMR (24\% CP, $25 \%$ fat, DM basis) and averaged $5.36 \mathrm{~g} / \mathrm{dL}$.

Diet by Week Effects. Interactions of diet by week for plasma glucose $(P=0.044)$ and plasma TG $(P=$ 0.001 ) concentrations were observed (Figure $2 \mathrm{~A}$ and B). In general, glucose concentration decreased with age, as expected. At the beginning of the trial, fasting plasma glucose averaged $98 \mathrm{mg} / \mathrm{dL}$ for all diets; at the end of the trial, glucose concentrations averaged 76 $\mathrm{mg} / \mathrm{dL}$. In calves on pSWM, glucose changed over time in a complex pattern [Figure 2A; significant linear $(P=$ $0.001)$ and quadratic $(P=0.008)$ contrast effects]. All calves fed CMR experienced linear decreases in glucose over time $(P=0.001$ for $100: 00,80: 20$, and $60: 40$, respectively; Figure 2A).

As with glucose, the diet by week interaction was significant for serum TG $(P=0.001)$. In calves fed pSWM, fasting serum TG decreased in a linear manner $(P=0.001)$ from 0 to $9 \mathrm{wk}$ (Figure 2B). In calves fed
100:00, TG changed over time in a complex pattern [Figure 2B; significant linear $(P=0.001)$ and quadratic $(P=0.001)$ contrast effects]. Calves fed 80:20 and $60: 40$ showed quadratic $(P=0.001$ and $P=0.001$, respectively) changes in TG from 0 to 9 wk (Figure 2B). Concentrations of TG in blood are indicative of LCFA absorbed from the diet that are incorporated into chylomicrons or from TG synthesized by and secreted from the liver. Here, calves fed pSWM most often had numerically higher fasting serum TG concentration than calves fed 80:20; this likely corresponds with the fact that calves fed pSWM ingested more total fat and, specifically, more total LCFA (Table 3). Another possibility is that clotting in the abomasum affects serum TG appearance in and clearance from the blood. To that point, Petit et al. (1988), fed Holstein calves either a clotting CMR or a nonclotting CMR (both $25 \% \mathrm{CP}$, $20 \%$ fat, DM basis), and noticed that calves fed clotting CMR had more uniform concentrations of plasma TG throughout a 24-h period, measured when calves were approximately 2-wk-old. Calves fed the nonclotting CMR showed plasma TG peaks $2 \mathrm{~h}$ postfeeding, with a decline thereafter (Petit et al., 1988).

Diet Effects. Fasting serum NEFA $(P=0.007)$ and PUN $(P=0.003)$ concentrations were affected by diet. Irrespective of week, calves fed 80:20 had higher serum NEFA concentrations than calves fed pSWM (0.28 vs. $0.21 \mathrm{mmol} / \mathrm{L} \pm 0.02$, respectively; $P=0.022$; Figure $2 \mathrm{C})$. The higher fasting NEFA concentrations in calves fed 80:20, as compared with those fed pSWM is in agreement with results from Bascom et al. (2007), where Jer- 

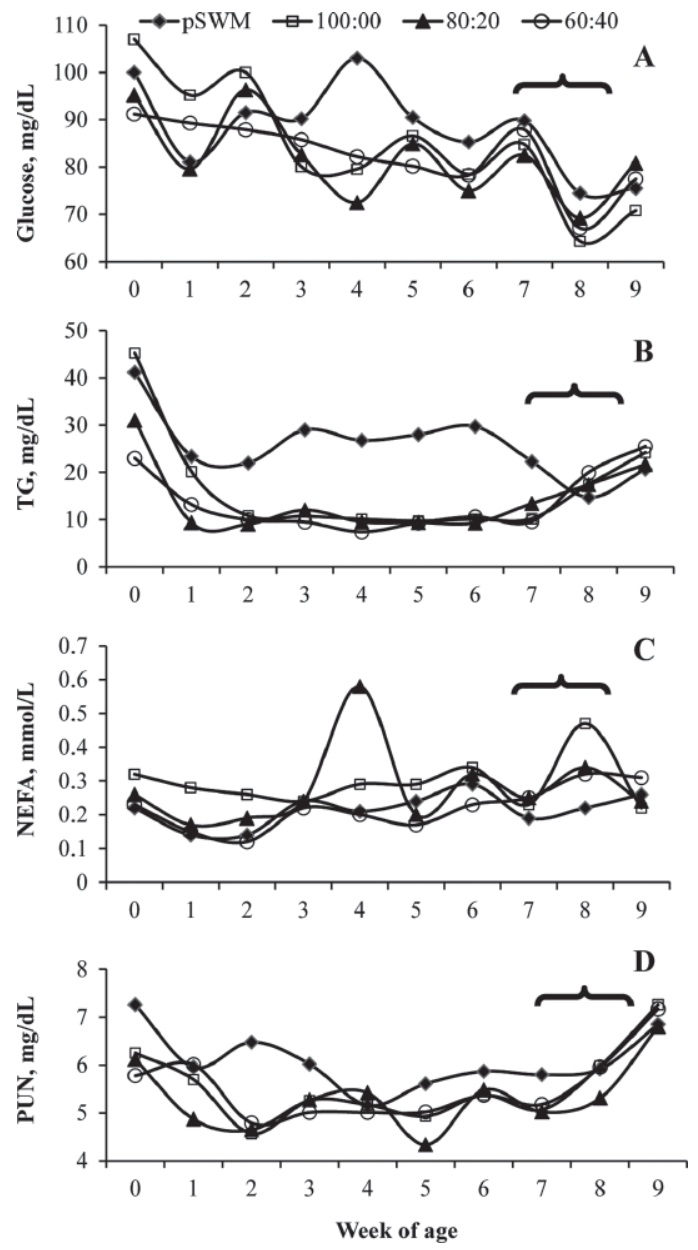

Figure 2. (A) Weekly 12-h fasting glucose; (B) triglyceride (TG); (C) NEFA; (D) and plasma urea nitrogen (PUN) concentrations in Jersey calves fed 1 of 4 diets. Diets were pasteurized saleable whole milk (pSWM; $27.9 \%$ CP, 33.5\% fat, DM basis); calf milk replacer (CMR; Cow's Match-Jersey Blend, Land O'Lakes Animal Milk Products, Shoreview, MN) containing $100 \%$ of fat as edible lard (100:00; $29.3 \%$ CP, $29.1 \%$ fat); CMR containing $20 \%$ of fat as coconut oil (CO; $80: 20$; $28.2 \%$ CP, $28.0 \%$ fat); and CMR containing $40 \%$ of fat as CO (60:40; $28.2 \% \mathrm{CP}, 28.3 \%$ fat). Weaning began at $7 \mathrm{wk}$ of age; calves averaged 8.2 wk of age at weaning (weaning period bracketed); weaning age did not differ by diet. A. The diet by week interaction was significant for glucose $(P=0.044)$; pSWM was linear $(P=0.001)$ and quadratic $(P=0.008)$ over time; and 100:00, 80:20, and 60:40 were linear over time $(P=0.001,0.001$, and 0.001 , respectively $)$. B. The diet by week interaction was significant for TG $(P=0.001)$; pSWM was linear $(P$ $=0.001)$ over time; 100:00 was linear $(P=0.001)$ and quadratic $(P$ $=0.001)$ over time; and 80:20 and 60:40 were quadratic over time $(P$ $=0.001$ and 0.001 , respectively). C. No interaction observed between diet and week for NEFA $(P=0.225)$. The main effect of diet was significant $(P=0.007)$; NEFA concentration differed between pSWM and 80:20 $(P=0.022)$. Coconut oil inclusion had a linear $(P=0.010)$ but not quadratic $(P=0.344)$ effect on NEFA concentrations. A main effect of week for NEFA $(P=0.005)$ was also observed. Regardless of diet, NEFA concentrations were linear $(P=0.015)$, but not quadratic $(P=0.225)$, over time. D. No interaction observed between diet and week for PUN $(P=0.945)$. The main effect of diet was significant $(P$ $=0.003) ;$ PUN concentration differed between pSWM and 80:20 $(P$ $=0.001)$. Coconut oil inclusion did not affect PUN concentration. A main effect of week for PUN $(P=0.001)$ was also observed. Regardless of diet, PUN concentrations were quadratic $(P=0.001)$, but not linear $(P=0.075)$, over time sey calves tended to have higher NEFA concentrations (2 h postfeeding) when fed a CMR (27\% CP; $33 \%$ fat; DM basis) compared with those fed SWM. Although measured NEFA can be from the ingestion of dietary FA, the 0600-h blood samples obtained here were after a 12-h fast, making it highly unlikely that measured NEFA came directly from dietary FA. This may suggest that calves fed 80:20 were in mild negative energy balance at the time of sample collection, and mobilized FA from body stores to meet energy needs before the next feeding. However, it should be noted that calves fed 80:20 grew the same as those fed pSWM (Table 2).

Irrespective of week, $\mathrm{CO}$ inclusion in the diet caused a linear $(P=0.010)$ decrease in NEFA concentrations (Figure 2C). Calves fed 100:00 had the highest NEFA values $(0.294 \mathrm{mmol} / \mathrm{L})$, followed by 80:20 (0.279 $\mathrm{mmol} / \mathrm{L})$, and $60: 40(0.220 \mathrm{mmol} / \mathrm{L}$; Figure $2 \mathrm{C})$. We expected that NEFA concentrations would increase as CO inclusion increased due to SCFA and MCFA being directly absorbed into the circulatory system; however, this is not what we observed and is likely be related to time of sample collection (after an overnight fast) in calves sampled at $0600 \mathrm{~h}$. It is interesting to speculate why this liner effect of CO inclusion was observed. Others have noted relative increases in serum NEFA with CMR feeding when edible lard is the primary fat source (Tikofsky et al., 2001; Bartlett et al., 2006; Bascom et al., 2007). Perhaps replacing edible lard with increasing amounts of $\mathrm{CO}$ alters fat metabolism to be more similar to the metabolism of milk fat, which contains more MCFA and less LCFA than edible lard. Though not one of our preplanned contrasts, the reader can see that the pattern of fasting NEFA concentration over 9 wk is similar in calves fed 60:40 and pSWM (Figure 2C).

Irrespective of week, calves fed pSWM had higher PUN concentration than calves fed 80:20 (6.09 \pm 0.15 vs. $5.33 \pm 0.15 \mathrm{mg} / \mathrm{dL}$, respectively; $P=0.001$; Figure 2D). This is likely due to the inherent MUN content of pSWM and the low amount of NPN in CMR. These findings are in agreement with research done by Górka et al. (2011), where it was shown that Holstein or Holstein $\times$ Limousine calves fed SWM had higher PUN concentrations before the morning feeding when compared with cohorts fed CMR (22\% CP; $17.5 \%$ fat; DM basis). As expected, CO inclusion did not affect PUN (Figure 2D).

Week Effects. An effect of week was observed for both fasting serum NEFA $(P=0.005)$ and PUN concentrations $(P=0.001)$. Irrespective of diet, NEFA concentrations increased linearly $(P=0.015)$ by week, with the lowest concentration observed at 2 wk $(0.18$ $\mathrm{mmol} / \mathrm{L})$ and the highest concentration at $8 \mathrm{wk}(0.34$ $\mathrm{mmol} / \mathrm{L}$; Figure 2C). Irrespective of diet, PUN concentrations were quadratic $(P=0.001)$ over time, with the 
highest concentrations observed at 0 and 9 wk $(6.35$ and $7.02 \mathrm{mg} / \mathrm{dL}$, respectively) and lowest at $5 \mathrm{wk}(4.97$ $\mathrm{mg} / \mathrm{dL}$; Figure 2D).

The first hypothesis of this study was that feeding CMR diets containing 0,20 , or $40 \%$ of total fat as CO to Jersey calves would result in either linear or quadratic effects on lipid deposition in the liver, gene expression in the liver, and blood metabolites. No changes in lipid content of the liver or liver gene expression were noted, so we failed to reject the null hypothesis that fat content of the liver and liver gene expression will be unaffected by the FA profile of the liquid feed. As for blood metabolites, for the vast majority of response variables measured we failed to reject our null hypothesis that blood metabolites will be unaffected by the FA profile of the liquid feed. Noted exceptions were with 12-h fasting plasma glucose, serum TG, and NEFA. Diet by week effects were noted for 12-h fasting plasma glucose and serum TG. Irrespective of week, inclusion of $\mathrm{CO}$ in the diet caused a linear decrease in 12-h fasting NEFA concentrations. This is interesting because a linear increase in total MCFA consumption with $\mathrm{CO}$ inclusion in CMR has been noted (Bowen Yoho et al., 2013). In general, lower NEFA concentrations are desirable in growing calves; the implications here are not clear but may merit further research with $\mathrm{CO}$ for this reason.

The second hypothesis was that feeding a CMR diet containing $20 \%$ of total fat as CO to Jersey calves would result in similar lipid deposition in the liver, gene expression in the liver, and blood metabolites when compared with Jersey calves fed pSWM. Liver lipid deposition and gene expression were not different between calves fed pSWM and calves fed 80:20. Some differences in 12-h fasting blood metabolites were noted between calves fed pSWM and calves fed 80:20. Effects attributed exclusively to diet were observed for serum NEFA and PUN. Irrespective of week, calves fed 80:20 had higher serum NEFA concentrations than calves fed pSWM. Calves fed pSWM higher PUN than 80:20, which the authors attribute to MUN in pSWM. The authors concede that comparisons between pSWM and 80:20 should be interpreted lightly because DMI from the liquid portion of the diet, as well as total fat intake were higher in calves fed pSWM, as a consequence of the experimental design.

\section{CONCLUSIONS}

In conclusion, for the vast majority of response variables measured we failed to reject our null hypotheses that lipid metabolism will be unaffected by the FA profile of the liquid feed. Our findings here, coupled with those noted in the companion paper by Bowen Yoho et al. (2013), seemingly provide evidence that MCFA in preruminant Jersey calf diets may not be as biologically important as we thought, with the possible exception being lower fasting serum NEFA concentrations with increased dietary MCFA.

\section{ACKNOWLEDGMENTS}

The authors express their sincere appreciation to Land O'Lakes Inc. Animal Milk Products Company (Shoreview, MN) for their generous donation of all the milk replacer used on this trial, as well as their financial support. In addition, we thank Purina Animal Nutrition LLC (St. Louis, MO) for providing the grain fed during this trial. Research support was also provided by state and federal funds appropriated to The Ohio State University, Ohio Agricultural Research and Development Center (\# 2011-120), and the American Jersey Cattle Association Research Fund (Reynoldsburg, OH; 2011). Lastly, we are grateful for the assistance provided by the Waterman Dairy Farm (The Ohio State University) staff during the trial.

\section{REFERENCES}

Bartlett, K. S., F. K. McKeith, M. J. Vandehaar, G. E. Dahl, and J. K. Drackley. 2006. Growth and body composition of dairy calves fed milk replacers containing different amounts of protein at two feeding rates. J. Anim. Sci. 84:1454-1467.

Bascom, S. A., R. E. James, M. L. McGilliard, and M. E. Van Amburgh. 2007. Influence of dietary fat and protein on body composition of Jersey bull calves. J. Dairy Sci. 90:5600-5609.

Bowen Yoho, W. S., V. A. Swank, M. L. Eastridge, K. M. O'Diam, and K. M. Daniels. 2013. Jersey calf performance in response to high protein, high fat liquid feeds with varied fatty acid profiles: Intake and performance. J. Dairy Sci. 96:2494-2506.

Daniels, K. M., S. R. Hill, K. F. Knowlton, R. E. James, M. L. McGilliard, and R. M. Akers. 2008. Effects of milk replacer composition on selected blood metabolites and hormones in preweaned Holstein heifers. J. Dairy Sci. 91:2628-2640.

DePeters, E. J., and J. D. Ferguson. 1992. Nonprotein nitrogen and protein distribution in the milk of cows. J. Dairy Sci. 75:31923209.

Górka, P., Z. M. Kowalski, P. Pietrzak, A. Kotunia, W. Jagusiak, and R. Zabielski. 2011. Is rumen development in newborn calves affected by different liquid feeds and small intestine development? J. Dairy Sci. 94:3002-3013.

Graulet, B., D. Gruffat-Mouty, D. Durand, and D. Bauchart. 2000. Effects of milk diets containing beef tallow or coconut oil on the fatty acid metabolism of liver slices from preruminant calves. Br. J. Nutr. 84:309-318.

Hocquette, J. F., and D. Bauchart. 1999. Intestinal absorption, blood transport and hepatic and muscle metabolism of fatty acids in preruminant and ruminant animals. Reprod. Nutr. Dev. 39:27-48.

Ikeda, Y., K. Okamura-Ikeda, and K. Tanaka. 1985. Purification and characterization of short-chain, medium-chain, and long-chain acyl-CoA dehydrogenases from rat liver mitochondria. isolation of the holo- and apoenzymes and conversion of the apoenzyme to the holoenzyme. J. Biol. Chem. 260:1311-1325.

Jenkins, K. J., J. K. G. Kramer, F. D. Sauer, and D. B. Emmons 1985. Influence of triglycerides and free fatty acids in milk replacers on calf performance, blood plasma, and adipose lipids. J. Dairy Sci. 68:669-680. 
Kahl, S., T. H. Elsasser, and J. W. Blum. 1997. Nutritional regulation of plasma tumor necrosis factor- $\alpha$ and plasma and urinary nitrite/ nitrate responses to endotoxin in cattle. Proc. Soc. Exp. Biol. Med. 215:370-376.

Lee, H. J., M. A. Khan, W. S. Lee, S. H. Yang, S. B. Kim, K. S. Ki, H. S. Kim, J. K. Ha, and Y. J. Choi. 2009. Influence of equalizing the gross composition of milk replacer to that of whole milk on the performance of Holstein calves. J. Anim. Sci. 87:1129-1137.

Loor, J. J., H. M. Dann, R. E. Everts, R. Oliveira, C. A. Green, N. A Guretzky, S. L. Rodriguez-Zas, H. A. Lewin, and J. K. Drackley. 2005. Temporal gene expression profiling of liver from periparturient dairy cows reveals complex adaptive mechanisms in hepatic function. Physiol. Genomics 23:217-226.

McFadden, J. W., and B. A. Corl. 2009. Activation of AMP-activated protein kinase (AMPK) inhibits fatty acid synthesis in bovine mammary epithelial cells. Biochem. Biophys. Res. Commun. 390:388-393.

Mills, J. K., D. A. Ross, and M. E. Van Amburgh. 2010. The effects of feeding medium-chain triglycerides on the growth, insulin responsiveness, and body composition of Holstein calves from birth to 85 $\mathrm{kg}$ of body weight. J. Dairy Sci. 93:4262-4273.

Morales, M. S., D. L. Palmquist, and W. P. Weiss. 2000. Effects of fat source and copper on unsaturation of blood and milk triacylglycerol fatty acids in Holstein and Jersey cows. J. Dairy Sci. 83:2105-2111.

Nguyen, P., V. Leray, M. Diez, S. Serisier, J. Le Bloc'h, B. Siliart, and H. Dumon. 2008. Liver lipid metabolism. J. Anim. Physiol. Anim. Nutr. (Berl.) 92:272-283.

Palmquist, D. L. 1988. The feeding value of fats. Page 293 in Feed Science. E. R. Orskov, ed. Elsevier Sci. Publ. A. V., Amsterdam, the Netherlands.
Petit, H. V., M. Ivan, and G. J. Brisson. 1988. Digestibility and blood parameters in the preruminant calf fed a clotting or a nonclotting milk replacer. J. Anim. Sci. 66:986-991.

Piot, C., J. F. Hocquette, J. H. Veerkamp, D. Durand, and D. Bauchart. 1999. Effects of dietary coconut oil on fatty acid oxidation capacity of the liver, the heart and skeletal muscles in the preruminant calf. Br. J. Nutr. 82:299-308.

Rinaldi, M., R. W. Li, D. D. Bannerman, K. M. Daniels, C. EvockClover, M. V. B. Silva, M. J. Paape, B. Van Ryssen, C. Burvenich, and A. V. Capuco. 2010. A sentinel function for teat tissues in dairy cows: Dominant innate immune response elements define early response to E. coli mastitis. Funct. Integr. Genomics 10:21-38.

Singh, I. 1997. Biochemistry of peroxisomes in health and disease. Mol. Cell. Biochem. 167:1-29.

Strable, M. S., and J. M. Ntambi. 2010. Genetic control of de novo lipogenesis: Role in diet-induced obesity. Crit. Rev. Biochem. Mol. Biol. 45:199-214.

Tikofsky, J. N., M. E. Van Amburgh, and D. A. Ross. 2001. Effect of varying carbohydrate and fat content of milk replacer on body composition of calves. J. Anim. Sci. 79:2260-2267.

United States Department of Agriculture, Animal Improvement Programs Laboratory. 2011. AIPL research report: State and national standardized lactation averages by breed for cows calving in 2010 . ARS:USDA:APIL. Beltsville, MD \#504.8334.

Zeng, J., and D. Li. 2005. Intrinsic isomerase activity of medium-chain acyl-CoA dehydrogenase. Biochemistry 44:6715-6722. 